\title{
Impaired Synergic Control of Posture in Parkinson's Patients without Postural Instability
}

\author{
Ali Falaki ${ }^{1}$, Xuemei Huang ${ }^{1,2,3,4,5}$, Mechelle M. Lewis ${ }^{1,2,3}$, Mark L. Latash ${ }^{1}$ \\ Department of Kinesiology ${ }^{1}$, The Pennsylvania State University, University Park, PA 16802 \\ Departments of Neurology ${ }^{2}$, Pharmacology ${ }^{3}$, Radiology ${ }^{4}$, and Neurosurgery ${ }^{5}$, Pennsylvania State \\ University-Milton S. Hershey Medical Center, Hershey PA 17033
}

Word count: 2998

Tables: 1

Figures: 2

Running Title: Postural synergies in Parkinson's disease

Address for correspondence:

Mark Latash

Department of Kinesiology

Rec.Hall-268N

The Pennsylvania State University

University Park, PA 16802

USA

Tel: (814) 863-5374

Fax: (814) 863-4424

E-mail: $\underline{\text { mll11@psu.edu }}$

Financial Disclosure/Conflict of Interest: No financial interest or conflict of interest is claimed by any of the authors.

Funding sources: XH and MML were supported by NIH grants NS060722, ES019672, and NS082151 during the past 12 months. MML also has an internal grant from Penn State University but received no salary support as part of the award. MLL and AF were supported by NIH grants NS035032 and AR048563.

$\underline{\text { Acknowledgment }}$

We would like to thank all the participants in the study. 
Abstract (200 words)

Background: Postural instability is one of most disabling motor symptoms in Parkinson's disease. Indices of multi-muscle synergies are new measurements of movement and postural stability.

Objectives: Multi-muscle synergies stabilizing vertical posture were studied in Parkinson's disease patients without clinical symptoms of postural instability (Hoehn-Yahr- $\leq$ II) and agematched controls. We tested the hypothesis that both synergy indices during quiet standing and synergy adjustments to self-triggered postural perturbations would be reduced in patients.

Methods: Eleven Parkinson's disease patients and 11 controls performed whole-body tasks while standing. Surface electromyography was used to quantify synergy indices stabilizing center of pressure shifts in the anterior-posterior direction during a load-release task.

Results: Parkinson's disease patients showed a significantly lower percentage of variance in the muscle activation space accounted for by the first four principal components, significantly reduced synergy indices during steady state, and significantly reduced anticipatory synergy adjustments (a drop in the synergy index prior to the self-triggered unloading).

Conclusions: The study demonstrates for the first time that impaired synergic control in Parkinson's disease can be quantified in postural tasks, even in patients without clinical manifestations of postural instability. Synergy measurements may provide a biomarker sensitive for early problems with postural stability in Parkinson's disease.

Key Words: Posture, Parkinson's disease, synergy, muscle mode, anticipatory synergy adjustments 


\section{INTRODUCTION}

Parkinson's disease (PD) is diagnosed clinically by the presence of rest tremor, rigidity, and bradykinesia. Postural instability and associated symptoms, such as episodes of freezing, emerge as the disease progresses and represent the most disabling symptoms of PD [1]. The presence of postural instability is a clinically important landmark in PD, signifying the transition from Hoehn-Yahr (HY, [2]) stage-II to stage-III. During typical clinical evaluations, postural instability is assessed using qualitative tests such as the pull test [3,4]. Whereas limitations of posturography in clinical studies have been emphasized recently [5], consistent changes in postural sway, postural adjustments prior to stepping, and responses to perturbations have been reported in stage-III PD [6-9].

The concept of motor synergy has evolved over the past 20 years (reviewed in $[10,11]$ ). Synergy is defined as a neural organization of a large set of effectors providing the stability of important performance variables [10]. For example, during multi-digit prehension, individual digit forces and moments co-vary to stabilize the resultant force/moment acting on the grasped object [12]. A synergy index has been introduced reflecting the relative amount of across-trials variance that does not affect a salient performance variable [10]. During steady-state actions, the synergy index typically is high. When a person is preparing for a quick action from a steady state, the synergy index drops $200-300 \mathrm{~ms}$ prior to action initiation. These anticipatory synergy adjustments (ASAs, $[13,14]$ ) represent an important reflection of controlled stability that allows combining stability during steady state and agility in transition to a quick action [15].

Recent studies in PD patients have suggested that indices of motor synergies may be used as sensitive biomarkers of PD even in extremities that show no clinically identifiable motor symptoms [16-18]. In particular, patients at HY stage-I (with clinical signs limited to one side of the body) showed comparably impaired indices of multi-finger synergies and impaired ASAs in both the symptomatic and asymptomatic hands [16].

In the current study, we explored whether indices of multi-muscle synergies stabilizing the coordinate of the center of pressure (COP) are able to detect postural stability changes in PD patients at $\leq$ HY stage-II without clinically identifiable postural instability. Our main hypothesis had two parts. First, during steady state (quiet standing) we expected lower synergy indices in PD patients compared to age-matched controls. Second, we expected significantly smaller ASAs in PD patients during preparation to a self-triggered postural perturbation. 


\section{METHODS}

\section{Subjects}

A group of 11 right-handed (6 females) PD patients without clinical postural instability (HY stage $\leq$ II, aged 69.4 \pm 6.3 years, mean \pm standard deviation, SD) and 11 (5 females) healthy controls (aged 65.3 \pm 8.1 years) participated in this study. Clinical postural stability was defined as lack of falls and negative on pull back test as part of Unified PD rating scale-part III. Detailed demographic and clinical information is presented in Table 1. All PD participants were tested on their prescribed medications. Written informed consent was obtained from all participants according to the protocol approved by the Penn State Hershey Institutional Review Board.

\section{Apparatus}

Subjects were tested while standing on a force platform (AMTI, OPTIMA). The platform recorded the horizontal component of the reaction force in the anterior-posterior direction $\left(\mathrm{F}_{\mathrm{X}}\right)$ as well as its vertical component $\left(\mathrm{F}_{\mathrm{Z}}\right)$, and the moment of force about a horizontal axis in the frontal plane $\left(\mathrm{M}_{\mathrm{Y}}\right)$. A 23" monitor mounted at eye level $1.5 \mathrm{~m}$ from the subject was used for visual feedback.

A 16-channel Trigno Wireless System (Delsys) was used to record the surface muscle activity (electromyogram, EMG). Active electrodes were placed over the bellies of the following right side muscles: tibialis anterior (TA), soleus (SOL), gastrocnemius medialis (GM), gastrocnemius lateralis (GL), biceps femoris (BF), semitendinosus (ST), rectus femoris (RF), vastus lateralis (VL), vastus medialis (VM), tensor fasciae latae (TFL), lumbar erector spinae (ESL), thoracic erector spinae (EST), and rectus abdominis (RA). EMG signals were preamplified and band-pass filtered $(20-450 \mathrm{~Hz})$ before being transmitted to the base station connected to a data collection computer (Dell, Xeon $2 \mathrm{GHz}$ ). EMG and force platform data were sampled at $1 \mathrm{KHz}$ with a 12-bit resolution (PCI-6225, National Instruments) using customized LabVIEW-based software (LabVIEW 2013).

\section{Procedures}

To ensure participant safety, all subjects used a safety harness. Initially, subjects were asked to stand on the force plate while keeping their feet parallel at shoulder width; the foot position was marked and reproduced across trials. The experiment started with a 30-s quiet 
standing trial used to record baseline EMG signals. The main experiment consisted of three tasks: voluntary sway (VS), fast-sway (FS), and load release (LR). Prior to each task, subjects performed a few familiarization trials.

VS task: The purpose of this task was to define a low-dimensional set of elemental variables (M-modes, muscle groups with parallel changes in activation levels [19]) and to link small changes in M-modes to $\mathrm{COP}_{\mathrm{AP}}$ shifts (the Jacobian, J [20,21], a matrix of partial derivatives of $\mathrm{COP}_{\mathrm{AP}}$ shifts to M-mode changes). Participants performed continuous voluntary body sway shifting $\mathrm{COP}_{\mathrm{AP}}$ between two targets shown on the screen over $6 \mathrm{~cm}$ at $0.5 \mathrm{~Hz}$ paced by a metronome. Two 30-s trials were performed.

FS task: The purpose of this task was to compare the ability to make fast whole-body actions between the groups. Subjects were asked to reach the posterior target $(3 \mathrm{~cm}$ away from the natural $\mathrm{COP}_{\mathrm{AP}}$ position), wait for $2 \mathrm{~s}$, and perform a very quick body sway forward to a target located $6 \mathrm{~cm}$ anterior. Twenty-four trials were performed.

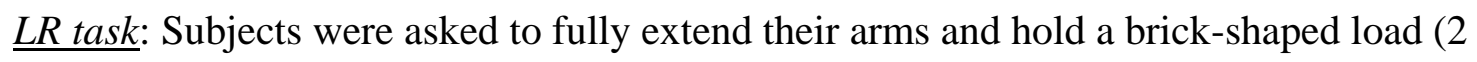
$\mathrm{kg}$ for males and $1.5 \mathrm{~kg}$ for females; $20 \mathrm{~cm}$ long) by pressing with palms on its sides. While holding the load, subjects were asked to shift their $\mathrm{COP}_{\mathrm{AP}}$ to a target $3-\mathrm{cm}$ anterior to the comfortable standing posture and to keep this position steadily for about 2-3 s. At a self-paced time, subjects released the load with a quick, low-amplitude bilateral shoulder abduction movement [22]. Subjects were instructed to avoid any further movement after releasing the load until the end of the trial. The task included 24 trials. Participants were provided with rest intervals (2-3-min after each 12 trials), snacks, and drinks as needed.

\section{Data Processing}

Recorded signals were processed offline using a customized MATLAB R2013a program. Force platform signals were low-pass filtered at $10 \mathrm{~Hz}$ with a fourth-order, zero-lag Butterworth filter. The filtered data were used to compute time-varying $\mathrm{COP}_{\mathrm{AP}}$ [23]. For the VS task, signals in the interval $\{3 \mathrm{~s} ; 28 \mathrm{~s}\}$ were considered for data analysis in order to avoid edge effects. On average, each participant performed ten full cycles within this period. During the FS and LR tasks, trials without major errors were accepted, $17 \pm 1$ for both groups.

\section{Basic EMG analysis}


Raw EMG signals were first band-pass filtered (20-350 Hz) using a fourth-order, zero-lag Butterworth filter, full-wave rectified and low-pass filtered by applying a moving average 100ms window. To account for the electro-mechanical delay [24], EMG data were shifted $50 \mathrm{~ms}$ backward with respect to the force platform data for computations involving both EMG and mechanical signals. EMG signals were corrected for baseline values and normalized $\left(\mathrm{IEMG}_{\mathrm{NORM}}\right)$ by the corresponding peak value across all trials.

Initiation of anticipatory postural adjustments (APAs $[25,26]$ ) in the LR task was defined using the time when the averaged across trials muscle activation level within a series performed by a subject exceeded \pm 2 SD from its respective mean activity (computed over the time interval $\left.\left\{\mathrm{T}_{0}-1000 \mathrm{~ms} ; \mathrm{T}_{0^{-}}-300 \mathrm{~ms}\right\}\right)$. GM, BF, ST, EST, and ESL muscles showed clear APAs in all participants (see Results); hence, the time when the earliest APA happened across these muscles was selected as $\mathrm{t}_{\mathrm{APA}}$ (confirmed visually).

\section{Defining muscle modes}

In our study, we assumed that individual muscles were united into groups, M-modes [18]. The first step identified such groups of muscles with co-varied scaling of activation levels. Principal component analysis (PCA) with factor extraction [19-21] after Varimax rotation was applied to the correlation matrix of the $\mathrm{IEMG}_{\mathrm{NORM}}$ data from the VS task integrated over 50-ms time windows. Based on the Kaiser criterion, four PCs were accepted and confirmed by visual inspection of the scree plot.

\section{Defining the Jacobian matrix}

The Jacobian matrix $(\mathbf{J})$ links small changes in the magnitude of M-modes $(\Delta \mathrm{M})$ to $\mathrm{COP}_{\mathrm{AP}}$ shifts $\left(\triangle \mathrm{COP}_{\mathrm{AP}}\right)$. $\mathbf{J}$ was defined by applying multiple linear regression analysis over all body sway cycles within the VS task:

$$
\triangle C O P_{A P}=k_{1} \Delta M_{1}+k_{2} \Delta M_{2}+k_{3} \Delta M_{3}+k_{4} \Delta M_{4}
$$

The coefficients of this multiple regression formed the $\mathbf{J}$ matrix: $\mathbf{J}=\left[k_{1} k_{2} k_{3} k_{4}\right]$. Prior to running the multiple linear regression analysis - to avoid effects unrelated to the body sway - a low-pass fourth-order, zero-lag Butterworth filter was used to filter $\Delta \mathrm{M}$ and $\Delta \mathrm{COP}_{\mathrm{AP}}$ at $5 \mathrm{~Hz}$.

\section{Analysis of synergies}


Synergies were analyzed according to previously published methods [20,21]. Briefly, under the framework of the uncontrolled manifold (UCM) hypothesis [27], synergies stabilizing $\mathrm{COP}_{\mathrm{AP}}$ shifts were quantified from the inter-trial variance in the muscle mode space to generate variance values that did not affect $\mathrm{COP}_{\mathrm{AP}}\left(\mathrm{V}_{\mathrm{UCM}}\right)$ and variance that did (variance in orthogonal directions, $\mathrm{V}_{\mathrm{ORT}}$ ). Computational details can be found in $[21,28]$. $\mathrm{V}_{\mathrm{UCM}}>\mathrm{V}_{\mathrm{ORT}}$ indicates a synergy stabilizing $\mathrm{COP}_{\mathrm{AP}}$ (reviewed in [10,29]). An index of synergy also was calculated: $\Delta \mathrm{V}=$ $\left(\mathrm{V}_{\mathrm{UCM}}-\mathrm{V}_{\mathrm{ORT}}\right) / \mathrm{V}_{\text {TOT }}$, where $\mathrm{V}_{\text {TOT }}$ is total variance and all variance indices are computed per dimension. For further statistical analysis, $\Delta \mathrm{V}$ was log-transformed using a modified Fisher's ztransform, $\Delta \mathrm{V}_{\mathrm{Z}}[30]$.

To quantify anticipatory synergy adjustments (ASAs), the synergy index was averaged within two time intervals during the LR task. The first was a 200-ms steady state ( $\mathrm{SS}_{1}$, Fig. 2A) from $\left(\mathrm{T}_{0}-1000\right) \mathrm{ms}$ to $\left(\mathrm{T}_{0}-800\right) \mathrm{ms}$, whereas the second was a $50-\mathrm{ms}$ time interval about $\mathrm{T}_{0}\left(\approx \mathrm{T}_{0}\right.$, Fig. 2A). ASA was quantified as the amount of $\Delta \mathrm{V}_{\mathrm{Z}}$ drop in preparation to the movement, $\Delta \Delta \mathrm{V}_{\mathrm{Z}}$.

\section{$\underline{\text { Statistics }}$}

Data are presented as means and standard errors (SE) in the text and figures. Two-way repeated measures ANOVA with factors Phase (two levels: $\mathrm{SS}_{0}$ and $\approx \mathrm{T}_{0}$ ) and Group (two levels: $\mathrm{PD}$ and controls) was performed to investigate possible differences in the synergy index $\left(\Delta \mathrm{V}_{\mathrm{Z}}\right)$ and its changes during ASAs. We also used t-tests and linear regression in the analysis of the Jacobian. For all analyses, the data were checked for violations of normality and sphericity. Geenhouse-Geisser correction was used to adjust the degrees-of-freedom when necessary. Pairwise contrasts with Bonferroni corrections were used to explore significant ANOVA effects. 


\section{RESULTS}

\section{Fast sway movement kinematics}

All subjects initiated the forward sway (FS) action with a brief shift backwards in the $\mathrm{COP}_{\mathrm{AP}}$. The magnitude of this shift did not differ between the groups, $1.5 \pm 0.28 \mathrm{~cm}$ in PD and $1.4 \pm 0.23 \mathrm{~cm}$ in controls. The overall magnitude of the forward COP shift also was similar between the groups $(10.3 \pm 0.35 \mathrm{~cm}$ and $10.3 \pm 0.53 \mathrm{~cm}$, respectively). There were no statistically significant differences between the peak rates of $\mathrm{COP}_{\mathrm{AP}}$ shift, $(\mathrm{dCOP} / \mathrm{dt})_{\mathrm{MAX}}$ being $38.3 \pm 3.11$ $\mathrm{cm} / \mathrm{s}$ and $42.0 \pm 5.91 \mathrm{~cm} / \mathrm{s}$ for the PD and controls, respectively.

\section{EMG patterns and anticipatory postural adjustments}

Averaged time profiles of normalized EMGs for a few selected leg and trunk muscles across LR trials performed by a representative PD subject are presented in Fig. 1. Most subjects showed consistent EMG profiles across trials, although muscle activation patterns were highly variable across subjects making across-subject data averaging meaningless. Significant EMG modulation was observed $\sim 140 \mathrm{~ms}$ before releasing the load $\left(\mathrm{T}_{0}\right)$, signifying anticipatory postural adjustments (APAs). APAs were observed in all subjects with GM, BF, ST, EST, and ESL muscles showing the most clear and reproducible APAs across participants. Arrows in Fig. 1 show $\mathrm{t}_{\mathrm{APA}}$ for the PD subject. There was no statistically significant group difference in $\mathrm{t}_{\mathrm{APA}}(-$ 138.3 $\pm 11.63 \mathrm{~ms}$ and $-140.4 \pm 8.86 \mathrm{~ms}$ for PD and controls, respectively).

\section{$<$ Figure 1 >}

\section{Defining M-modes and Jacobian}

On average, the first four M-modes accounted for less of the total variance in PD subjects compared to controls $\left(71.5 \pm 1.74 \%\right.$ vs. $\left.78.3 \pm 1.74 \% ; \mathrm{F}_{(1,20)}=7.969 ; \mathrm{p}<0.05\right)$. Multiple regression analysis of shifts in $\mathrm{COP}_{\mathrm{AP}}\left(\Delta \mathrm{COP}_{\mathrm{AP}}\right)$ as functions of changes in M-modes $(\Delta \mathrm{M}$-modes) showed that all M-modes were significant predictors of $\Delta \mathrm{COP}_{\mathrm{AP}}$ for each subject $(\mathrm{p}<0.001)$. The linear regression, on average, showed similar adjusted $R^{2}$ values of $0.66 \pm 0.03$ and $0.70 \pm 0.04$ for PDs and controls, respectively (no group difference). 


\section{Analysis of the synergy index}

During quiet standing $\left(\mathrm{SS}_{1}\right)$, the synergy index $\left(\Delta \mathrm{V}_{\mathrm{Z}}\right)$ was significantly lower in PD subjects compared to controls $(0.014 \pm 0.07$ vs. $0.22 \pm 0.06$; $\mathrm{p}<0.05$; see Fig. $2 \mathrm{~A})$. There was a larger drop in the synergy index in controls from steady state to the moment of load release (Phase $\times$ Group interaction; $\left.\mathrm{F}_{(1,20)}=9.84, \mathrm{p}<0.01\right)$. The magnitudes of the synergy index at the two time intervals studied are presented in Fig. 2B. Note that the synergy index drops in controls in preparation to action initiation (ASAs) and this is absent in the PD group.

$<$ Figures 2> 


\section{DISCUSSION}

To our knowledge, the current study demonstrates for the first time that studies of multi-muscle synergies during postural tasks are able to identify sub-clinical postural stability changes in PD patients. This finding suggests the potential of using multi-muscle synergy analysis to develop a quantitative biomarker able to identify issues with postural stability in patients with PD who show no postural instability during clinical examination.

\section{Synergies in PD}

The word "synergy" is used in the movement science literature in at least three different ways (reviewed in [31]). In the clinical literature, this word has a negative connotation and means stereotypical patterns of muscle activation that interfere with intentional movements $[32,33]$. In the motor control literature, "synergy" frequently means a number of performance variables that show parallel changes in task parameters or over time [34-37]. In particular, muscle activation analysis allows identifying muscle groups (M-modes) within which muscles show parallel activation changes. We define synergies as a neural organization of a set of Mmodes that ensures stability of a task-specific performance variable ( $\mathrm{COP}_{\mathrm{AP}}$ coordinate) by covariation of the M-mode involvement across repetitive trials [30].

A number of earlier studies documented synergic control changes in PD. Until now, those studies focused on finger coordination [16-18,38]. The current study extended these previous experiments to the synergic control of posture. Two aspects of changed synergic control were observed across systems (digits and muscles) and tasks (pressing, prehension, and vertical posture). All studies reported reduced $\Delta \mathrm{V}$ indices at steady states and lower ASAs in preparation to a quick action. The similarity of the findings across systems and tasks suggests a general impairment in the neural mechanisms involved in the control of stability of motor performance in PD. This makes the individual results specific reflections of a basic problem reviewed recently as impaired control of stability, ICS [15].

To our knowledge this is first study to test multi-muscle synergies in PD during a postural task. As hypothesized, PD patients showed a significantly smaller amount of variance in muscle activation that was accounted for by the first four M-modes. This observation suggests a less consistent organization of muscles into M-modes, possibly related to the sway task being 
perceived as more challenging by the PD group (cf. [39]). In addition, PD subjects showed significantly reduced indices of M-mode synergies stabilizing $\mathrm{COP}_{\mathrm{AP}}$. Note that we tested PD patients without clinically identifiable signs of postural instability. Early-stage PD is associated with measurable changes in gait and balance indices, which can even be observed in persons at high risk of PD [40,41]. These indices include, in particular, trunk sway measures [42]. Our study provides a complementary approach to early problems with postural control in PD based on the theory of synergic control. Future studies are warranted to follow the progression of postural synergic indices to investigate whether these measures may be able to predict future clinical postural instability.

\section{Impaired feed-forward control of synergies in PD}

We explored two aspects of feed-forward postural control, anticipatory postural adjustments, APAs [25,26] and anticipatory synergy adjustments, ASAs [13,28]. APAs typically are observed about $100 \mathrm{~ms}$ prior to the action/perturbation initiation. The purpose of APAs has been assumed to generate net forces and moments of force acting against those expected from the anticipated action/perturbation. PD patients with clinical postural instability (HY stage-III) show reduced and delayed APAs [43-45]. In our study, no significant difference in the APAs was observed between PDs and controls, a finding that is consistent with the lack of clinical postural instability in our PD patients.

Another aspect of feed-forward control is anticipatory synergy adjustments (ASAs [13]), which are seen as changes in the synergy index stabilizing a salient performance variable prior to a quick change in that variable. ASAs are seen as early as 250-350 ms prior to action initiation. Their purpose has been assumed to induce gradual destabilization in order to facilitate a planned quick change. We observed significantly reduced ASAs in PD subjects, suggesting that this aspect of feed-forward postural control may be more sensitive than APA to measure PD-related impairment. The documented reduction in ASAs in the PD group in the current study (as well as in earlier studies of hand function $[16,18,38]$ ) is a key finding and should be emphasized.

Without ASAs, the nervous system would have to fight its own synergies stabilizing a variable in a preceding steady state. Indeed, it is possible that some actions may be nearly impossible to initiate without ASAs, in particular those starting from a steady state where stability is essential. Vertical posture is an example of a steady-state task that serves as the background for many 
whole-body actions. Although speculative, an impaired ability to generate ASAs could be related causally to episodes of disabling freezing, which are common in later stages of PD.

\section{Concluding comments}

The most obvious limitation of our study is the relatively small size of the PD and control groups. Nevertheless, the current study provides the first evidence of impaired synergic control during postural tasks in PD and follow-up studies are warranted. It would be highly desirable to conduct a longitudinal follow-up study of these subjects and/or compare synergy indices across HY stages to evaluate the predictive value of synergy indices. In addition, it also would be important to compare indices of synergies with a broader range of clinical indices reflecting postural stability and freezing of gait such as postural sway and step/turn initiation in PD. 


\section{Authors' Roles}

Ali Falaki: Research Project (Organization, Execution); Statistical Analysis (Design, Execution), Manuscript Preparation (Writing of the first draft, Review and Critique)

Mechelle Lewis: Research Project (Organization); Statistical Analysis (Review and Critique), Manuscript Preparation (Review and Critique)

Xuemei Huang: Research Project (Conception, Organization); Statistical Analysis (Review and Critique), Manuscript Preparation (Review and Critique)

Mark Latash: Research Project (Conception Organization, Execution); Statistical Analysis (Design, Review and Critique), Manuscript Preparation (Writing of the first draft, Review and Critique)

\section{Financial Disclosure}

XH was supported by NINDS NS060722, NIEHS ES019672, NINDS NS082151, CIH-287375, GE Life Sciences, and Penn State Hershey internal funds during the past 12 months. XH has potential royalty interests in University owned IP. MML was supported by NINDS NS060722, NIEHS ES019672, and NINDS NS082151, and also has an internal grant from Penn State University but received no salary support as part of the award. MLL and AF were supported by NIH grants R01 NS035032 and R01 AR048563. 


\section{References}

1. Marras C, McDermott MP, Rochon PA, et al. Survival in Parkinson disease: thirteen-year follow-up of the DATATOP cohort. Neurology 2005; 64: 87-93.

2. Hoehn M, Yahr M. Parkinsonism: onset, progression and mortality. Neurology 1967; 17: 42742

3. Goetz CG, Tilley BC, Shaftman SR, et al. Movement Disorder Society-sponsored revision of the Unified Parkinson's Disease Rating Scale (MDS- UPDRS): scale presentation and clinimetric testing results. Mov Disord 2008; 23: 2129-2170.

4. Fahn S, Jankovic J. Principles and Practice of Movement Disorders. Churchill Livingstone, Philadelphia, PA, 2007.

5. Visser JE, Carpenter MG, van der Kooij H, Bloem BR. The clinical utility of posturography. Clin Neurophysiol 2008; 119: 2424-2436.

6. King LA, St George RJ, Carlson-Kuhta P, Nutt JG, Horak FB. Preparation for compensatory forward stepping in Parkinson's disease. Arch Phys Med Rehab 2010; 91: 1332-1338.

7. Nardone A, Schieppati M. The role of instrumental assessment of balance in clinical decision making. Eur J Phys Rehabil Med. 2010; 46: 221-237.

8. Rogers MW, Kennedy R, Palmer S, et al. Postural preparation to stepping in patients with Parkinson's disease. J Neurophysiol 2011; 106: 915-924.

9. Boonstra TA, van Vugt JPP, van der Kooij H, Bloem BR. Balance asymmetry in Parkinson's disease and its contribution to freezing of gait. PLoS One 2014; 9: e102493.

10. Latash ML, Scholz JP, Schöner G. Toward a new theory of motor synergies. Motor Control 2007; 11: 276-308.

11. Latash ML. The bliss (not the problem) of motor abundance (not redundancy). Exp Brain Res. 2012; 217:1-5.

12. Zatsiorsky VM, Latash M.L. Multi-finger prehension: An overview. J Mot Behav 2008; 40: 446-476.

13. Olafsdottir H, Yoshida N, Zatsiorsky VM, Latash ML. Anticipatory covariation of finger forces during self-paced and reaction time force production. Neurosci Lett 2005; 381: 92-96.

14. Shim JK, Olafsdottir H, Zatsiorsky VM, Latash ML. The emergence and disappearance of multi-digit synergies during force production tasks. Exp Brain Res. 2005; 164: 260-270. 
15. Latash ML, Huang X. Neural control of movement stability: Lessons from studies of neurological patients. Neuroscience 2015; 301: 39-48.

16. Park J, Wu Y-H, Lewis MM, Huang X, Latash ML. Changes in multi-finger interaction and coordination in Parkinson's disease. J Neurophysiol 2012; 108: 915-924.

17. Park J, Jo HJ, Lewis MM, Huang X, Latash ML. Effects of Parkinson's disease on optimization and structure of variance in multi-finger tasks. Exp Brain Res 2013; 231: 51-63.

18. Park J, Lewis MM, Huang X, Latash ML. Dopaminergic modulation of motor coordination in Parkinson's disease. Parkinsonism Rel Disord 2014; 20: 64-68.

19. Krishnamoorthy V, Goodman SR, Latash ML, Zatsiorsky VM. Muscle synergies during shifts of the center of pressure by standing persons: Identification of muscle modes. Biol Cybern 2003; 89: 152-161.

20. Danna-Dos-Santos A, Slomka K, Zatsiorsky VM, Latash ML. Muscle modes and synergies during voluntary body sway. Exp Brain Res. 2007; 179: 533-550.

21. Krishnamoorthy V, Latash ML, Scholz JP, Zatsiorsky VM. Muscle synergies during shifts of the center of pressure by standing persons. Exp Brain Res. 2003; 152: 281-292.

22. Aruin AS, Latash ML. The role of motor action in anticipatory postural adjustments studied with self-induced and externally-triggered perturbations. Exp Brain Res 1995; 106: 291-300.

23. Winter DA, Prince F, Frank JS, et al. Unified theory regarding A/P and M/L balance in quiet stance. J Neurophysiol 1996; 75: 2334-2343.

24. Corcos DM, Gottlieb GL, Latash ML, et al. Electromechanical delay: An experimental artifact. J Electromyogr Kinesiol 1992; 2: 59-68.

25. Belenkiy VY, Gurfinkel VS, Paltsev YI. Elements of control of voluntary movements. Biofizika 1967; 10: 135-141.

26. Massion J. Movement, posture and equilibrium - interaction and coordination. Prog Neurobiol 1992; 38: 35-56.

27. Scholz JP, Schöner G. The uncontrolled manifold concept: Identifying control variables for a functional task. Exp Brain Res. 1999; 126, 289-306.

28. Klous M, Mikulic P, Latash ML. Two aspects of feed-forward postural control: Anticipatory postural adjustments and anticipatory synergy adjustments. J Neurophysiol 2011; 105: 22752288. 
29. Latash ML, Scholz JP, Schöner G. Motor control strategies revealed in the structure of motor variability. Exer Sport Sci Rev 2002; 30: 26-31.

30. Solnik S., Pazin N., Coelho C., et al. End-state comfort and joint configuration variance during reaching. Exp Brain Res. 2013; 225: 431-442.

31. Latash ML. Synergy. Oxford Univ. Press: NY, 2008.

32. Bobath B. Adult Hemiplegia: Evaluation and Treatment. William Heinemann, London, 1978.

33. DeWald JP, Pope PS, Given JD, et al. Abnormal muscle coactivation patterns during isometric torque generation at the elbow and shoulder in hemiparetic subjects. Brain 1995; 118: 495-510.

34. d'Avella A, Saltiel P, Bizzi E. Combinations of muscle synergies in the construction of a natural motor behavior. Nat Neurosci 2003; 6: 300-308.

35. Ivanenko YP, Poppele RE, Lacquaniti F. Five basic muscle activation patterns account for muscle activity during human locomotion. J Physiol 2004; 556: 267-282.

36. Ting LH, Macpherson JM. A limited set of muscle synergies for force control during a postural task. J Neurophysiol 2005; 93: 609-613.

37. Tresch MC, Cheung VC, d'Avella A. Matrix factorization algorithms for the identification of muscle synergies: evaluation on simulated and experimental data sets. J Neurophysiol 2006; 95: 2199-212.

38. Jo HJ, Park J, Lewis MM, Huang X, Latash ML. Prehension synergies and hand function in early-stage Parkinson's disease. Exp Brain Res 2015; 233: 425-440.

39. Danna-Dos-Santos A, Degani AM, Latash ML. Flexible muscle modes and synergies in challenging whole-body tasks. Exp Brain Res. 2008; 189: 171-187.

40. Baltajieva R, Giladi N, Gruendlinger L, et al. Marked alterations in the gait timing and rhythmicity of patients with de novo Parkinson's disease. Eur J Neurosci 2006; 24: 1815 1820.

41. Maetzler W, Hausdorff JM. Motor signs in the prodromal phase of Parkinson's disease. Mov Disord 2012; 27: 627-633.

42. Maetzler W, Mancini M, Liepert-Scarfone I, et al. Impaired trunk stability in individuals at high risk for Parkinson's disease. PLoS One 2012; 7: e32240. 
43. Bazalgette D, Zattara M, Bathien N, et al. Postural adjustments associated with rapid voluntary arm movements in patients with Parkinson's disease. Adv Neurol 1986; 45: 371374.

44. Dick J.P.R., Rothwell J.C., Berardelli A., et al. Associated postural adjustments in Parkinson's disease. J Neurol Neurosurg Psychiatry 1986; 49: 1378-1385.

45. Diener HC, Dichgans J, Guschlbauer B, Bacher M, Langenbach P. Disturbances of motor preparation in basal ganglia and cerebellar disorders. Prog Brain Res 1989; 80: 481-488. 
Table 1 Description of the participants

\begin{tabular}{|c|c|c|c|c|c|c|}
\hline \multicolumn{7}{|c|}{ PD group } \\
\hline Patient & $\begin{array}{l}\text { Gender, } \\
\mathrm{M} / \mathrm{F}\end{array}$ & $\begin{array}{l}\text { Age, } \\
\text { years }\end{array}$ & $\begin{array}{l}\text { Symptom } \\
\text { Onset, R/L }\end{array}$ & $\begin{array}{l}\text { Years since } \\
\text { diagnosis }\end{array}$ & $\begin{array}{l}\text { UPDRS motor } \\
\text { score }\end{array}$ & $\begin{array}{l}\text { Total LEDD, } \\
\mathrm{mg}\end{array}$ \\
\hline 1 & $\bar{F}$ & 72 & $\bar{R}$ & 3.5 & 11 & 300 \\
\hline 2 & $\mathrm{~F}$ & 73 & $\mathrm{~L}$ & 4.5 & 18 & 480 \\
\hline 3 & $\mathrm{~F}$ & 72 & $\mathrm{~L}$ & 4 & 38 & 300 \\
\hline 4 & $\mathrm{~F}$ & 70 & Bilateral & 0.8 & 13 & 500 \\
\hline 5 & M & 72 & $\mathrm{R}$ & 4.6 & 15 & 167 \\
\hline 6 & M & 69 & Bilateral & 1.6 & 8 & 195 \\
\hline 7 & M & 79 & $\mathrm{R}$ & 2.2 & 8 & 500 \\
\hline 8 & M & 67 & $\mathrm{~L}$ & 7.6 & 5 & 737.5 \\
\hline 9 & M & 71 & $\mathrm{R}$ & 3.1 & 21 & 400 \\
\hline 10 & $\mathrm{~F}$ & 55 & $\mathrm{R}$ & 2.2 & 2 & 250 \\
\hline 11 & $\mathrm{~F}$ & 63 & $\mathrm{R}$ & 4.7 & 18 & 700 \\
\hline Mean & $\mathrm{F}(6)$ & $67.5 \pm 7.4$ & & & & \\
\hline Mean & $\mathrm{M}(5)$ & $71.6 \pm 4.6$ & & & & \\
\hline \multicolumn{7}{|c|}{$\mathrm{CO}$ group } \\
\hline \multicolumn{2}{|c|}{ Control } & \multicolumn{3}{|c|}{ Gender, M/F } & \multicolumn{2}{|l|}{ Age, years } \\
\hline \multirow{2}{*}{\multicolumn{2}{|c|}{1}} & \multicolumn{3}{|l|}{$\mathrm{M}$} & \multicolumn{2}{|l|}{56} \\
\hline & & \multicolumn{3}{|l|}{$\mathrm{F}$} & \multicolumn{2}{|l|}{58} \\
\hline \multicolumn{2}{|l|}{3} & \multicolumn{3}{|l|}{$\mathrm{F}$} & \multicolumn{2}{|l|}{67} \\
\hline \multicolumn{2}{|l|}{4} & \multicolumn{3}{|l|}{$\mathrm{F}$} & \multicolumn{2}{|l|}{54} \\
\hline \multirow{2}{*}{\multicolumn{2}{|c|}{5}} & \multicolumn{3}{|l|}{ M } & \multicolumn{2}{|l|}{77} \\
\hline & 6 & \multicolumn{3}{|l|}{ M } & \multicolumn{2}{|l|}{78} \\
\hline \multicolumn{2}{|l|}{7} & \multicolumn{3}{|l|}{ M } & \multicolumn{2}{|l|}{69} \\
\hline \multirow{2}{*}{\multicolumn{2}{|c|}{8}} & \multicolumn{3}{|l|}{$\mathrm{F}$} & \multicolumn{2}{|l|}{71} \\
\hline & & \multicolumn{3}{|l|}{ M } & 64 & \\
\hline \multicolumn{2}{|l|}{$\begin{array}{l}9 \\
10\end{array}$} & M & & & 63 & \\
\hline 11 & & F & & & 61 & \\
\hline Mean & & $\mathrm{F}(5)$ & & & $62.1 \pm 7.1$ & \\
\hline Mean & & M (6) & & & $68.0 \pm 8.4$ & \\
\hline
\end{tabular}

M/F: male/females, R/L: right/left, LEDD: levodopa equivalent daily dose, UPDRS: Unified Parkinson's Disease Rating Scale. 


\section{Figure Captions}

\section{Figure 1}

Normalized EMG time profiles of a few selected muscles across the load-release (LR) trials for a representative PD subject: tibialis anterior (TA), soleus (SOL), gastrocnemius medialis (GM), biceps femoris (BF), rectus femoris (RF), and thoracic erector spinae (EST). The solid lines are the averages of the corresponding muscle activity across trials while the shaded areas shows one standard error. The vertical dashed lines represent the moment when the subject released the load. Arrows in the EMG panels mark the onset of APAs ( $\left.\mathrm{t}_{\mathrm{APA}}\right)$.

\section{Figure 2}

A: Time profiles of the synergy index $\left(\Delta \mathrm{V}_{\mathrm{Z}}\right)$, averaged across patients (dashed line) and controls (solid line), during the load-release (LR) task. $\mathrm{SS}_{1}$ and $\approx \mathrm{T}_{0}$ are the time intervals selected for statistical analysis. Note the significantly higher $\Delta \mathrm{V}_{\mathrm{Z}}$ in $\mathrm{CO}$ and the drop in this index before $T_{0}$. B: Z-transformed synergy indices $\left(\Delta \mathrm{V}_{\mathrm{Z}}\right)$ for the PD (black bar) and CO (white bar) groups over the $\mathrm{SS}_{1}$ and $\approx \mathrm{T}_{0}$ phases. Mean $\pm \mathrm{SE}$ values across subjects within each group are shown. Statistically significant differences are marked with a star. 

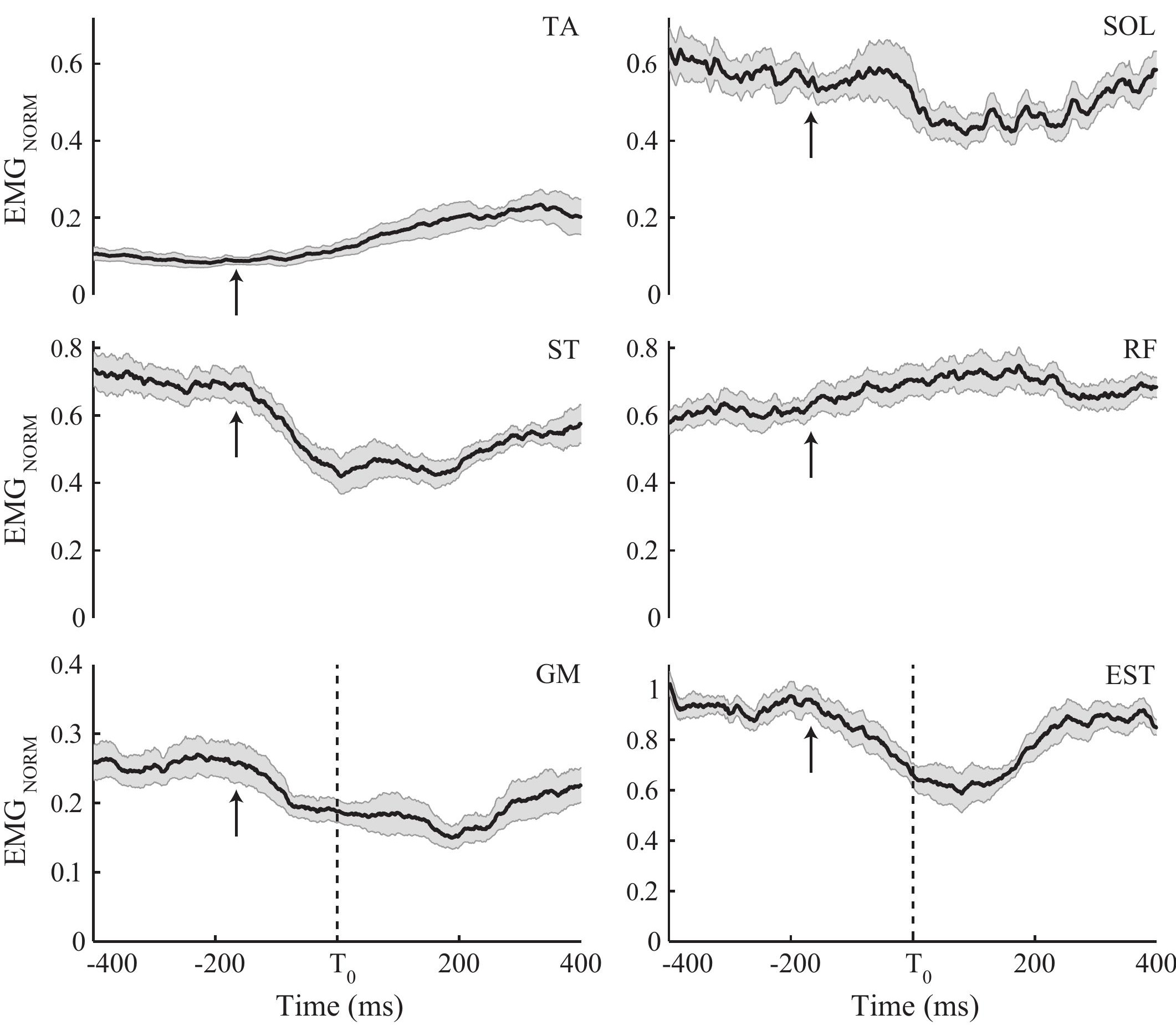
